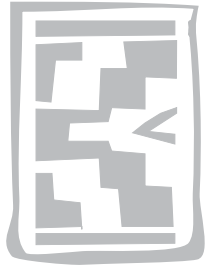

\title{
Intramammary antibiotics in dairy goats: withdrawal periods of three intramammary antibiotics compared to recommended withdrawal periods for cows
}

\author{
J. KARZIS ${ }^{1 *}$, E.F. DONKIN² and I.M. PETZER ${ }^{1}$
}

\begin{abstract}
KARZIS, J., DONKIN, E.F. \& PETZER, I.M. 2007. Intramammary antibiotics in dairy goats: withdrawal periods of three intramammary antibiotics compared to recommended withdrawal periods for cows. Onderstepoort Journal of Veterinary Research, 74:217-222

Intramammary antibiotics are registered and tested for use in dairy cattle. This study investigated withdrawal periods of three intramammary antibiotics (Curaclox LC [Norbrook Pharmacia AH]), Spectrazol Milking Cow (Schering-Plough Animal Health) and Rilexine 200 LC (Logos Agvet [Virbac]) in dairy goats and compared them to withdrawal periods recommended for use in cattle. Three trials were carried out in two different herds. The withdrawal periods for Curaclox LC in eight relatively low producing Saanen goats were $74.3 \mathrm{~h}( \pm 19.21)$ measured by Thermo-Resistant Inhibitory Substances (TRIS), $90.0 \mathrm{~h}( \pm 16.97)$ measured by colour dye, $99.4 \mathrm{~h}( \pm 9.07)$ for cloxacillin measured by Parallux Beta Lactam Assay test (IDEXX distributors), and $92.6 \mathrm{~h}( \pm 11.41)$ for ampicillin measured by Parallux. The withdrawal period for Curaclox LC recommended for use in cattle $(72 \mathrm{~h})$ was significantly shorter than the withdrawal periods as measured by colour dye $(P<0.001)$, Parallux testing for cloxacillin $(P<0.001)$ and ampicillin $(P=0.003)$. There was a significant difference in withdrawal periods as measured by TRIS $(P=0.009)$ and colour dye $(P=0.036)$. The mean withdrawal periods measured on 12 relatively high producing Saanen and Saanen-Toggenburg crossbreed dairy goats measured by TRIS, colour dye and Parallux for ampicillin and cloxacillin were, however, shorter at $42.0 \mathrm{~h}( \pm 7.077)$, $64.5 \mathrm{~h}( \pm 60.26), 77.3 \mathrm{~h}( \pm 13.56)$ and $70.7 \mathrm{~h}( \pm 12.65)$, respectively. These withdrawal periods for Curaclox LC were significantly longer than the withdrawal periods recommended for cattle as measured by TRIS $(P<0.001)$ and colour dye $(P<0.001)$. The combined withdrawal periods for Curaclox LC in the two trials as measured by TRIS, colour dye, Parallux testing for Cloxacillin and Ampicillin were 58.64h ( \pm 24.31$), 75.8 \mathrm{~h}( \pm 17.70), 87.0 \mathrm{~h}( \pm 16.10)$ and $80.3 \mathrm{~h}( \pm 16.23)$, respectively. The withdrawal period, when data of the two trials were combined, measured by TRIS $(P<0.001)$ was significantly longer than the withdrawal period recommended for use in cattle. The mean withdrawal period as measured by TRIS for Spectrazol Milking Cow for seven relatively low producing Saanen dairy goats was $95.3 \mathrm{~h}( \pm 17.23)$. This was significantly $(P<0.001)$ longer than the withdrawal period recommended for use in cattle $(60 \mathrm{~h})$ for Spectrazol Milking Cow.
\end{abstract}

The mean withdrawal period as measured by TRIS for Rilexine 200 LC for 20 relatively high producing Saanen and Saanen-Toggenburg crossbreed dairy goats was $36.9 \mathrm{~h}( \pm 9.943)$ and was significantly $(P<0.001)$ shorter than the withdrawal period as recommended for use in cattle $(96 \mathrm{~h})$.

There were also significant differences in all the one-sample t-tests between withdrawal periods for goats and recommended withdrawal periods for cattle without the $24 \mathrm{~h}$ safety margin.

Keywords: Antibiotic withdrawal periods, Curaclox LC (Norbrook [Pharmacia AH]), dairy goats, Parallux, Rilexine 200 LC (Logos Agvet [Virbac]), Spectrazol Milking Cow (ScheringPlough Animal Health), TRIS

* Author to whom correspondence is to be directed. E-mail: jkarzis@mweb.co.za or joannejk@ iafrica.com

1 Department of Production Animal Studies, Faculty of Veterinary Science, University of Pretoria, Private Bag X04, Onderstepoort, 0110 South Africa
2 Department of Animal and Wildlife Sciences, Faculty of Natural and Agricultural Sciences, University of Pretoria, 0002 South Africa

Accepted for publication 19 March 2007-Editor 


\section{INTRODUCTION}

The demand for goat milk is steadily increasing, due to the production of specific cheeses from goat milk (Jaubert \& Kalantzopoulos 1996) and the use of goat milk for children who are intolerant to other sources of milk (Fisberg, Ferreira, Noguiera \& Fisberg 2000). Dairy goats, rather than cows, are often more appropriate for subsistence milk production (Donkin \& Boyazoglu 2000).

One of the most threatening diseases to dairy goats is mastitis (Smith \& Sherman 1994). At present, mastitis in goats is treated with antibiotics used for treating bovine mastitis. Initially assumptions were made that withdrawal times for antibiotic residues in milk are the same for goats as for cows (Debackere 1995). However, limited research has shown that residues persist for a longer period in goat milk than in milk from cows (Bangen, Skjerve, Grave \& Soli 1992). The milk may not be used during and after mastitis treatment, due to the long, probable withdrawal times of antibiotic residues in goat milk. Antibiotic residues in goat milk may pose a serious health hazard for humans consuming goat milk, especially babies, as anaphylactic and allergic reactions may occur due to these residues, as well as the danger of development of resistant strains of bacteria.

The production of cheese is also seriously affected by the presence of antibiotic residues in the milk. Therefore, the consequences of mastitis and antibiotic treatment lead to economic losses in the goat dairy farming industry (Smith \& Sherman 1994). Thus, research is necessary to determine the most appropriate antibiotic preparations to use for the treatment of mastitis in goats as well as to establish appropriate withdrawal times for these preparations.

This research will assist in management of dairy goats in developing communities and also for commercial farmers. Milk is an ideal supplement to reduce malnutrition (Malentlema 1987) and can have a significant impact on livestock development projects (Pelant 2000).

\section{MATERIALS AND METHODS}

\section{Herds used in trials}

Three experimental trials were conducted. Trial 1 and Trial 2 were conducted at The Faculty of Veterinary Science, University of Pretoria (Herd A), while Trial 3 was conducted on a commercial goat dairy in the Limpopo Province of South Africa (Herd B).
Trial 1

Herd A consisted of 15 lactating multiparous Saanen goats and was conducted over a period of 7 days using 14 goats, all except one of which were in early lactation. Trial 1 commenced in winter with low temperatures and no rainfall.

\section{Trial 2}

Trial 2 was conducted in Herd A over a period of 8 days using 14 lactating multiparous Saanen goats. The Trial took place in spring with moderate temperatures and with light rainfall in the evenings. Goats were in mid lactation, except for one that was in late lactation.

\section{Trial 3}

Trial 3 was conducted over a period of 8 days in Herd B, using 64 Saanen, Saanen/indigenous crossbreeds and Toggenburg goats, all of which were in mid lactation. Of the 64 goats, 57 were in their first and the rest in their second lactation. Trial $3 \mathrm{com}$ menced in summer with high temperatures, high humidity and low rainfall.

In all trials, results of clinical udder examinations, milk production, age and stage of lactation were evaluated and selection of experimental animals was done by the principle of pairing. Daily milk production was categorized as low $(<1.3 \ell)$, medium (1.3$1.5 \ell$ ) and high $(>1.5 \ell$ ).

Goats were considered "infected", when bacteria were isolated from the milk sample.

\section{Sampling in all trials}

Goats were milked at 12 hourly intervals and foremilk samples were taken aseptically from udder halves of all goats according to the procedure of (Giesecke, Du Preez \& Petzer 1994). Two sets of udder half milk samples and a composite sample were taken during three milkings prior to treatment to measure baseline parameters. Udder halves were milked separately and milk volumes were recorded. Sampling continued until the Somatic Cell Counts (SCC) returned to baseline values and at least two consecutive Thermo-Resistant Inhibitory Substances (TRIS) test results were negative for all experimental animals.

\section{Antibiotic treatment}

\section{Products investigated}

The three intramammary preparations used are available as commercial formulations for the treatment of 
bovine mastitis. Each contains the required dose of antibiotics in a suitable substrate and tube for direct administration into the udder via the teat canal.

In Trial 1, six untreated goats were used as controls, while eight goats were treated with Curaclox LC (Norbrook [Pharmacia AH]), $75 \mathrm{mg}$ sodium ampicillin and $200 \mathrm{mg}$ sodium cloxacillin per dose. In Trial 2 , seven goats were untreated controls and seven goats were treated, after each of three consecutive milkings at 12 hourly intervals, with Spectrazol Milking Cow (Schering-Plough Animal Health), $250 \mathrm{mg}$ cefuroxime per dose. Two products were investigated in Trial 3: Rilexine (SA) 200 LC (Logos Agvet [Virbac]), $100 \mathrm{mg}$ cephalexin, $100 \mathrm{mg}$ neomycin sulphate and $10 \mathrm{mg}$ prednisolone per dose, and Curaclox LC. Twenty untreated goats acted as controls and 20 goats were treated, after each of three consecutive milkings at 12 hourly intervals, with Rilexine (SA) 200 LC, while 12 goats were treated with Curaclox LC and 12 goats served as controls.

\section{Administration of antibiotics}

The entire content of the intramammary antibiotic syringe was inserted into each udder half of the goats in the treatment groups after three consecutive milkings at 12 hourly intervals.

\section{Laboratory procedures}

Laboratory procedures were conducted at the milk laboratory (Department of Production Animal Studies, Faculty of Veterinary Science, University of Pretoria).

\section{Visual inspection of milk samples}

The colours of the samples treated with Curaclox LC, containing the blue dye, were matched to colours on the Pasco Colour Expressions colour chart in order to standardize the different variations in the colour of the blue dye in goat milk. This was carried out at every subsequent milking after intramammary treatment.

\section{Thermo-resistant inhibitory substances (TRIS)}

The TRIS test was carried out on all milk samples to determine the presence of antibiotic residues and other heat stable inhibitory substances in milk (Karzis 2005).

\section{The Parallux ${ }^{\mathrm{TM}}$ Beta Lactam Assay Test}

The Parallux ${ }^{\mathrm{TM}}$ Beta Lactam Assay (IDEXX distributors) is a competitive solid-phase fluorescence im- munoassay intended for use as a rapid detection method for Pen* (penicillin G, amoxicillin, ampicillin, cloxacillin, ceftiofur and cephapirin) residues in raw commingled bovine milk. The Veterinary Diagnostic Services Laboratory, North West Province performed this analysis.

Milk samples of 16 of the goats with the longest withdrawal periods in Trials 1 and 3 that were treated with Curaclox LC were selected to determine the difference in the withdrawal period for ampicillin and cloxacillin. Only milk from the left udder halves was tested.

All samples were frozen immediately after they were taken. The samples were subsequently thawed and diluted 50:50 with distilled water and mixed well before use. This dilution factor was necessary for the samples to reach the correct viscosity, so that the result could be read by the Parallux machine.

\section{Data management}

All data were entered and stored in Microsoft Excel and data were statistically analysed using the computer software GenStat (2003).

\section{RESULTS}

\section{Statistical analysis of withdrawal periods for goats compared to withdrawal periods recommended for use in cattle with or without the $24 \mathrm{~h}$ safety margin}

Table 1 showed withdrawal periods obtained in Trials 1, 2 and 3 for Curaclox LC, Spectrazol and Rilexine compared to withdrawal periods recommended for use in cattle.

\section{Curaclox LC from Trials 1 and 3 combined}

Table 2 showed withdrawal periods measured by different methods for Curaclox LC in Trials 1 and 3.

\section{DISCUSSION}

\section{Withdrawal periods of intramammary antibiotics for goats compared to withdrawal periods recommended for use in cattle with or without the $24 \mathrm{~h}$ safety margin}

The withdrawal period of a particular commercially available preparation recommended for use in cattle has a $24 \mathrm{~h}$ safety margin added to the longest withdrawal period obtained in the trial for the preparation. In this study this $24 \mathrm{~h}$ safety margin has not 
Intramammary antibiotics in dairy goats: withdrawal periods

TABLE 1 Withdrawal period (WP) of intramammary antibiotics of goats compared to withdrawal periods (WPs) recommended for use in cattle with or without the $24 \mathrm{~h}$ safety margin (one sample t-tests)

\begin{tabular}{|c|c|c|c|c|}
\hline $\begin{array}{l}\text { Trials and products } \\
\text { used }\end{array}$ & $\begin{array}{l}\text { WPs measured by } \\
\text { different methods }\end{array}$ & $\begin{array}{l}\text { Mean } \pm \text { Standard } \\
\text { Deviation }(h) \text { WP } \\
\text { recommended for } \\
\text { use in cattle }\end{array}$ & $\begin{array}{l}\text { F probability WP } \\
\text { recommended for } \\
\text { use in cattle }\end{array}$ & $\begin{array}{l}\text { F probability WP } \\
\text { recommended for } \\
\text { use in cattle }-24 \mathrm{~h}\end{array}$ \\
\hline Trial 2; Spectrazol & WP TRIS & $95 \pm 17.23$ & $P<0.001$ & $P<0.001$ \\
\hline Trial 3; Rilexine & WP TRIS & $37 \pm 9.943$ & $P<0.001$ & $P<0.001$ \\
\hline $\begin{array}{l}\text { Trials } 1 \text { and } 3 \text {; } \\
\text { Curaclox LC }\end{array}$ & $\begin{array}{l}\text { WP TRIS } \\
\text { WP Colour Dye } \\
\text { WP Cloxacillin Parallux } \\
\text { WP Ampicillin Parallux }\end{array}$ & $\begin{array}{l}59 \pm 24.31 \\
76 \pm 17.70 \\
87 \pm 16.10 \\
80 \pm 16.23\end{array}$ & $\begin{array}{l}P<0.0018 \\
P=0.160 \\
P=0.002 \\
P=0.060\end{array}$ & $\begin{array}{l}P=0.006 \\
P<0.001 \\
P<0.001 \\
P<0.001\end{array}$ \\
\hline Trial 1; Curaclox LC & $\begin{array}{l}\text { WP TRIS } \\
\text { WP Colour Dye } \\
\text { WP Cloxacillin Parallux } \\
\text { WP Ampicillin Parallux }\end{array}$ & $\begin{array}{l}74 \pm 19.21 \\
90 \pm 16.97 \\
99 \pm 9.07 \\
93 \pm 11.41\end{array}$ & $\begin{array}{l}P=0.646 \\
P<0.001 \\
P<0.001 \\
P=0.003\end{array}$ & $\begin{array}{l}P<0.001 \\
P<0.001 \\
P<0.001 \\
P<0.001\end{array}$ \\
\hline Trial 3; Curaclox LC & $\begin{array}{l}\text { WP TRIS } \\
\text { WP Colour Dye }\end{array}$ & $\begin{array}{l}42 \pm 7.08 \\
65 \pm 7.76\end{array}$ & $\begin{array}{l}P<0.001 \\
P<0.001\end{array}$ & $\begin{array}{l}P<0.001 \\
P<0.001\end{array}$ \\
\hline
\end{tabular}

$P$ is significant at the $5 \%$ level $(P<0.05)$

TABLE 2 Withdrawal periods (WPs) measured by different methods (Curaclox LC; Trials 1 and 3)

\begin{tabular}{|l|l|l|l|}
\hline Variate & Sample size & Mean \pm Standard Deviation (h) & Probability \\
\hline Difference between WP TRIS and WP Colour Dye & 44 & $17 \pm 464.8$ & $P<0.001$ \\
Difference between WP TRIS and WP Cloxacillin Parallux & 16 & $26 \pm 13.30$ & $P<0.001$ \\
Difference between WP TRIS and WP Ampicillin Parallux & 16 & $20 \pm 13.77$ & $P<0.001$ \\
\hline
\end{tabular}

been added and, in practice, a $24 \mathrm{~h}$ safety margin should be added to all the withdrawal periods determined in this investigation. The raw data from the trials carried out on cattle are not available. Therefore, by subtracting the $24 \mathrm{~h}$ safety margin from the withdrawal period recommended for use in cattle an estimated withdrawal period was obtained for cows.

This estimated withdrawal period for cows was also compared to the withdrawal periods of intramammary antibiotics found in goats. These data are shown in Table 1. There were significant differences in all the one-sample t-tests between withdrawal periods for goats compared to withdrawal periods recommended for use in cattle (without the $24 \mathrm{~h}$ safety margin). Not all were significant when compared with withdrawal periods as recommended for use in cattle (Table 1).

\section{Withdrawal periods of Curaclox LC as measured by different methods compared to withdrawal periods recommended for use in cattle}

Zeng, Escobar \& Brown-Crowder (1996) showed that the withdrawal period according to the Penzyme Test, the Delvotest $\mathrm{P}$ and the TRIS test are $72 \mathrm{~h}$ for
Penicillin $\mathrm{G}$ and $120 \mathrm{~h}$ for Cephapirin. In cattle the TRIS test is routinely used for the detection of antibiotic residues. A negative TRIS test is an indication of the maximum residue limits (MRLs) and safe tolerance levels for antibiotic residues in milk fixed by Codex Alimetarius, EC-Regulations and United States Food and Drug Administration (USFDA). The MRLs European Community (EC) for ampicillin, cloxacillin, cephalosporins and neomycin are $4 \mu \mathrm{g} / \mathrm{kg}$, $30 \mu \mathrm{g} / \mathrm{kg}, 100 \mu \mathrm{g} / \mathrm{kg}$ and $500 \mu \mathrm{g} / \mathrm{kg}$, respectively and those in the MRLs Codex for ampicillin, cloxacillin, cephalosporins and neomycin are $4 \mu \mathrm{g} / \mathrm{kg}, 4 \mu \mathrm{g} / \mathrm{kg}$, $100 \mu \mathrm{g} / \mathrm{kg}$, and $500 \mu \mathrm{g} / \mathrm{kg}$, respectively (HonkanenBuzalski \& Reybroeck 1997). The Parallux test was more sensitive for testing goat milk for antibiotic residues than the TRIS test, but it was much more expensive. Combined data of Trials 1 and 3 for Curaclox LC showed significantly shorter withdrawal periods $(P<0.001)$ measured by TRIS $(59 \mathrm{~h})$ and significantly longer $(P=0.002)$ withdrawal periods for cloxacillin $(87 \mathrm{~h})$ measured by Parallux compared to the $72 \mathrm{~h}$ recommended for use in cattle. The mean withdrawal period determined by the colour dye (76 h) and Parallux for ampicillin ( $80 \mathrm{~h}$ ) did not differ significantly from that recommended for use in cattle. 
During Trial 1 low milk producers were used as experimental animals to test Curaclox LC. The mean withdrawal period as measured by TRIS ( $74 \mathrm{~h}$ ) during this trial did not differ significantly from that recommended for use in cattle. However, results obtained in the same trial from the colour dye $(90 \mathrm{~h})$ and the Parallux measurements for cloxacillin (99 h) and ampicillin $(93 \mathrm{~h})$ are all significantly $(P<0.001)$ longer than those recommended for use in cattle (Table 1).

During Trial 3 high milk producers were used as experimental animals to test Curaclox LC. In this trial the mean withdrawal period, as measured by TRIS $(42 \mathrm{~h})$ and colour dye $(65 \mathrm{~h})$, is significantly $(P<$ 0.001 ) shorter than that recommended for use in cattle. In the same Trial the mean withdrawal period, as measured by the Parallux testing for both cloxacillin and ampicillin does not differ significantly from that recommended for use in cattle (Table 1).

Cloxacillin residues appeared to remain longer than the ampicillin residues (Table 1), but this is not a significant difference. However, the withdrawal periods as measured by the Parallux for both ampicillin and cloxacillin residues differ significantly $(P<0.001)$ from the withdrawal periods measured by the TRIS test (Table 2). Differences between withdrawal periods as measured by TRIS and colour dye are also highly significant $(P<0.001)$ (Table 2$)$.

The withdrawal period as measured by TRIS of relatively high producers $(>1.5 \ell)$ in Trial 3 is significantly shorter than that $(74 \mathrm{~h})$ obtained for low producers $(<1.3 \ell)$ in Trial 1 , after both had been treated with Curaclox LC. This is also shown by the strong negative correlation between volume and withdrawal period in Trial $1\left(R^{2}=-0.701\right)$ (Karzis 2005). There is no significant difference in withdrawal periods between left and right udder halves (inter-half variance) as measured by different methods for all three products used (Karzis 2005).

\section{Withdrawal periods of Spectrazol Milking Cow and Rilexine 200 LC as measured by different methods compared to withdrawal periods recommended for use in cows}

Withdrawal periods in goats measured by TRIS proved to be significantly $(P<0.001)$ longer $(95 \mathrm{~h})$ than those recommended for use in cows $(60 \mathrm{~h})$. Antibiotics can be more dependent on concentration or more dependent on time for efficacy. Bacteriostatic antibiotics and beta-lactams are time dependant.

Rilexine 200 LC contains cephalexin, which is time dependent, and neomycin, which is concentration dependent. The withdrawal period for Rilexine 200 LC in goats in Trial 3 is significantly $(P<0.001)$ shorter than the $96 \mathrm{~h}$ recommended for use in cows. The goats treated with Rilexine 200 LC were relatively high producers.

In this investigation it is evident that the volume of milk produced had an effect on the withdrawal period and that high producers $(>1.5 \ell)$ had shorter withdrawal periods than low producers $(<1.3 \ell)$.

\section{Differences in withdrawal periods between infected and non-infected udder halves treated with Curaclox LC, Spectrazol Milking Cow and Rilexine 200 LC}

The withdrawal periods measured after intramammary treatment with Curaclox LC in the combined data of Trials 1 and 3 do not differ significantly with any of the three tests used (TRIS, colour dye and Parallux) between infected and non-infected udder halves (Karzis 2005), nor were any significant differences found in withdrawal periods of infected and non-infected udder halves during Trial 1 (Karzis 2005). However, the mean withdrawal period as measured by TRIS is significantly different $(P=$ $0.024)$ at $5 \%$ level, between infected ( $40 \mathrm{~h}$ ) and noninfected $(47 \mathrm{~h})$ udder halves during Trial 3 in goats treated with intramammary Curaclox LC.

There is no significant difference in Trial 2 after treatment with Spectrazol Milking Cow in the mean withdrawal period as measured by the TRIS test, between infected (96 h) and non-infected (95 h) udder halves. Therefore the presence of bacteria prior to treatment did not affect the length of the withdrawal period.

The difference between mean withdrawal periods as measured by the TRIS test for infected ( $38 \mathrm{~h}$ ) and non-infected $(27 \mathrm{~h})$ udder halves differ significant at the $5 \%$ level $(P=0.034)$ in Trial 3 after the intramammary administration of Rilexine 200 LC.

\section{CONCLUSION}

Antibiotic withdrawal periods for goat milk differed depending on the test used for that determination. The Parallux test proved to be more sensitive than the TRIS test and the colour dye tests for Curaclox LC, while the dye test remained positive for longer than the TRIS test. After Curaclox LC treatment in goats, significantly shorter withdrawal periods than those recommended for cattle were found with the TRIS test in Trial 3 and the combined data of Trial 1 and 3 , while the results obtained in Trial 3 (relatively 
high producers) showed no significant difference. The colour dye test was positive for a significantly longer period in Trial 1 and shorter period in Trial 3 , compared to the recommended withdrawal period for cows. The Parallux withdrawal periods proved to be significantly longer in Trial 1 (relatively low producers) and the combined data of Trials 1 and 3 , while in Trial 3 (relatively high producers) they did not differ significantly from the withdrawal period recommended for cows.

Significant differences were found between withdrawal periods for goats compared to withdrawal periods recommended for use in cows without the $24 \mathrm{~h}$ (safety margin), but not all of these tests are significant when the comparison is made with withdrawal periods as recommended for use in cows (including the $24 \mathrm{~h}$ safety margin).

The withdrawal period for Spectrazol Milking Cow is significantly longer and that for Rilexine 200 LC is shorter than the respective recommended withdrawal periods in cows. Withdrawal periods were affected by the milk volume produced by the goats. Low producers $(<1.3 \ell)$ had a longer withdrawal period than relatively high producers $(>1.5 \ell)$, treated with the same intramammary antibiotic.

There is no significant difference in withdrawal periods between infected and non-infected udder halves treated with Curaclox LC in Trial 1 and the combined data of Trial 1 and 3 and with Spectrazol Milking Cow. However, significantly longer withdrawal periods were found in infected udder halves treated with Rilexine 200 LC and for goats with non-infected udder halves treated with Curaclox LC in Trial 3.

Further investigations are required to determine MRLs and safe/tolerance levels of antibiotic residues specifically for goat milk and in addition, the most reliable test for achieving these results needs to be determined.

\section{ACKNOWLEDGEMENTS}

We thank the National Research Foundation for the funding of this research, Mr Tobie Fourie and the staff of the Limpopo Melkery, the staff of the Milk Laboratory, Onderstepoort, the staff of the Onderstepoort Teaching Animal Unit for all their help and Marie Smith of the Agricultural Research Council, Silverton for the statistical analysis.

\section{REFERENCES}

BANGEN, M., SKJERVE, E., GRAVE, K. \& SOLI, N.E. 1992. Prescribing of drugs for food producing animals in Norway. Information about withdrawal times. Journal of Veterinary Pharmacology and Therapeutics, 15:180-187.

DEBACKERE, M. 1995. Pharmacokinetics and pharmacodynamics of antimicrobials in relation to their residues in milk. Proceedings of a Symposium on Residues of Antimicrobial Drugs and other Inhibitors in Milk, Kiel, Germany, 1995: 41.

DONKIN, E.F. \& BOYAZOGLU, P.A. 2000. Milk production from goats for households and small-scale farmers in South Africa. Proceedings of the $7^{\text {th }}$ International Conference on Goats, Tours, France, 2000: 324-326.

FISBERG, M., FERREIRA, A.M.A., NOGUIERA, M. \& FISBERG, R.M. 2000. Acceptance and tolerability of goat milk in preschool children. Proceedings of the th International Conference on Goats, Tours, France, 2000: 881-883.

GenStat $₫$ for Windows $₫$ 2003. Introduction, edited by R.W. Payne. VSN International.

GIESECKE, W.H., DU PREEZ, J.H. \& PETZER, I.M. 1994. Practical mastitis control in dairy herds. Durban: Butterworths: 93-111.

HONKANEN-BUZALSKI, T. \& REYBROECK, W. 1997. Residues and contaminants in milk and milk products: Antimicrobials. International Dairy Federation Special Issue 9701:26-28

JAUBERT, G. \& KALANTZOPOULOS, G. 1996. Quality of goat milk for cheese and other products. Proceedings of the $6^{\text {th }}$ International Conference on Goats, Beijing, China, 1996: 274-281.

KARZIS, J. 2005. Intramammary antibiotics in dairy goats: Withdrawal periods and tissue tolerance. M.Sc. thesis, University of Pretoria.

MALENTLEMA, T.N. 1987. Nutritional aspects of milk products in developing countries, in Milk-the vital force, edited by The Organizing Committee of the XXII International Dairy Congress, D. Reidel Publishing Company: 269-277.

PELANT, R.K. 2000. Approaches to goat-related projects for economic development. Proceedings of the $7^{\text {th }}$ International Conference on Goats, Tours, France, 15-18 May 2000: 3-8.

SMITH, M.C. \& SHERMAN, D.M. 1994. Goat medicine. Baltimore, Maryland, USA: Lippincott, Williams \& Wilkins: 465-494.

ZENG, S.S., ESCOBAR, E.N. \& BROWN-CROWDER, I. 1996. Evaluation of screening tests for detection of antibiotic residues in goat milk. Small Ruminant Research, 21:155-160. 